\title{
Modeling of droplet dynamic and thermal behaviour during spray deposition
}

\author{
N S MAHESH*, JOHNSON MENDONCA, M K MURALIDHARA, B K MURALIDHARA ${ }^{\dagger}$ \\ and C RAMACHANDRA \\ Department of Mechanical Engineering, M.S. Ramaiah Institute of Technology, Bangalore 560 054, India \\ ${ }^{\dagger}$ Department of Mechanical Engineering, University Visvesvaraya College of Engineering, Bangalore 560 001, India \\ ${ }^{\star}$ Department of Chemical Engineering, Fenn College of Engineering, Cleveland, OH 44115, USA
}

MS received 3 October 2002; revised 7 January 2003

\begin{abstract}
Mathematical modeling of supersonic gas atomization for spray forming has been investigated. Influence of the droplet dynamic and thermal behaviour on the resultant microstructure has been studied. Analytical models have been constructed taking into account the higher Reynolds number owing to supersonic gas flow. The impact velocity profiles of the droplets lend credence to the evolution of equiaxed grain morphology through dendrite fragmentation. The thermal history profile along with the fraction solid plot could yield optimized standoff distance to obtain a mushy droplet. A comparison of secondary dendrite arm spacing obtained from the mathematical model showed good agreement with experimental observations.
\end{abstract}

Keywords. Supersonic atomization; droplets; thermal history; solid fraction; secondary dendrite arm spacing.

\section{Introduction}

Spray deposition refers to disintegration of a molten metal stream into variously sized droplets. These droplets are propelled away from the atomization region by fast flowing atomizing gas to a substrate/platform where they collect and coalesce to form a solid preform (Gutierrez et al 1988; Lavernia et al 1988; Ojha 1992; Grant 1995; Lawley 2000). The two major stages in spray casting are spray atomization and spray deposition. The spray atomization stage consists of the following aspects: Highly energetic gas jet or jets (supersonic velocity) impinge on a stream of molten metal leading to break up of the stream into small, irregular ligaments. The spherodization of small, irregular ligaments occurs leading to formation of spherical droplets. Since a sphere has the least surface area for a given volume and as a result minimum surface energy (product of surface tension and surface area), spherical droplets are formed. It has been well established that the spherodization is completed almost immediately after the formation of small, irregular ligaments since most of the molten metals have high surface tension. The droplets sizes will be in a wide range depending on the atomization variables. It has been reported that the size distribution of the droplets is lognormal (Lubanska 1970; Unal 1987, 1988; Bewlay and Cantor 1990; Grant et al 1993; Grant 1995). These droplets will be entrained in a spray cone. The momentum transfer occurs from atomizing gas to droplets, which governs the droplet velocity, the flight

*Author for correspondence time and subsequent deformation behaviour during the deposition stage. The droplets lose their heat to atomizing gas during the flight and solidify completely or partially depending on their size and velocity. The next stage in spray casting is collection of these droplets on a substrate or platform to result in a deposit or preform.

The microstructure of the final deposit is related to the state of the droplets impinging on the top surface of the growing preform. In order to know the physical state of the droplets at the time of impingement, thermal history analysis of the droplets is inevitable. The atomized droplets lose heat mostly by convection due to the surrounding gas. The droplets experience different cooling rates/ heat transfer rates depending on their size and internal crystallization nuclei. The gas atomization may lead to (a) fully solid droplet, (b) fully liquid droplet with a temperature above the liquidus, (c) partially solidified droplet with a temperature between the liquidus and solidus and (d) fully liquid droplet with a temperature below the liquidus (undercooled droplet) (Gutierrez et al 1989). The spray can contain the droplets with sizes between 20 and 500 micrometers and hence the largest droplets will be in conditions such as (b) and (c) while the smallest droplets can be in conditions such as (a) and (d) (Perepezko and Park 1982). During atomization, the heat transfer rates are extremely high in the order of $10^{3}$ to $10^{6} \mathrm{~K} / \mathrm{s}$ and hence spray casting comes under rapid solidification processes (Lavernia et al 1988; Mathur et al 1989; Lavernia 1989; Grant and Cantor 1991; Grant et al 1993).

It has been shown that the final spray formed microstructure and yield efficiency are strongly dependent on overall solid fraction in the spray upon impact with the 
substrate (Mathur et al 1989; Gutierrez et al 1989). Further, it has been reported that (Grant et al 1993; Eon-Sik Lee and Ahn 1994; Grant and Cantor 1995) the solid fraction impinging on the billet top surface is too low, splashing and whipping up of liquid material by the gas produces pores of entrapped gas in the final billet; excessive solid fraction reaching the surface can also give porous billet since insufficient molten metal is available to flow over the billet to fill pores and interstices. The overall solid fraction depends on process variables viz. atomizing gas pressure, gas to metal ratio, melt superheat, stand-off distance and alloy composition (Eon-Sik Lee and Ahn 1994). In essence, it is important to understand droplet dynamic and thermal behaviour to quantify the effect of the above mentioned process variables on the evolution of microstructure in spray formed billets.

This paper reports an attempt made to analyse the droplet velocities and heat transfer in the flow field from the exit of the nozzle to the substrate. The mathematical model developed for computing the thermal histories (Szekely 1979; Lavernia et al 1988; Mathur et al 1989; Gutierrez et al 1989; Grant et al 1993; Eon-Sik Lee and Ahn 1994; Dimos and John 1997) was reviewed and improvised to account for higher Reynolds number encountered in the present situation. With regard to dynamic behaviour of the droplets, velocity profile of droplets of different sizes was plotted. The output from the above was used to analyse the thermal behaviour of droplets. In this connection, fall in temperature of the variously sized droplets and also their quench rates as they traverse the distance from the atomization region to the substrate were calculated. Further, solid fraction in the droplets as a function of flight distance was computed and finally secondary dendrite arm spacing for different droplet sizes was predicted.

\section{Model formulation}

The dynamic behaviour of the droplets is a function of gas velocity decay and droplet size. In order to compute the droplet dynamic behaviour, gas velocity variation as a function of flight distance has to be plotted. Further, the velocity of droplets of various sizes was to be found based on force balance. The objective of computing droplet velocity is to calculate the velocity of impact on the substrate and cooling rate, which influences the microstructure.

Cooling and solidification behaviour of droplets take place in five different thermal regions (Mathur et al 1989; Eon-Sik Lee and Ahn 1994) viz. liquid phase cooling, recalescence, segregated solidification, eutectic solidification and solid phase cooling. In the present study, the cooling of droplets up to recalescence temperature has been considered and cooling rate for later stages has been extrapolated. It is well established that recalescence occurs in a time interval of the order of microseconds and most of the solidification takes place under a constant cooling rate for all droplet sizes encountered in high pressure gas atomization (Lavernia et al 1988). In the light of above, a constant cooling rate region of the cooling rate plot was considered for predicting SDAS for a given droplet size.

\subsection{Gas and droplet velocities}

Knowledge of the gas flow field generated by the gas atomizer is a prerequisite for characterizing the transport phenomenon controlling droplet dynamics and heat transfer during the droplet flight. Although gas flow behaviour is a critical factor in the spray forming process, there is no available analytical solution of the gas jet issuing from the atomizing nozzle (Eon-Sik Lee and Ahn 1994). However, a good deal of experimental work has been reported (Bewlay and Cantor 1989; Anderson et al 1991; $\mathrm{Xu}$ and Lavernia 2000) on gas velocity measurements and flow mechanism in high-pressure gas atomization.

During gas atomization, a liquid stream of metal is disintegrated into droplets by the impact of an energetic gas jet. The gas velocity is maximum at exit from the atomizer nozzle and subsequently decreases with distance as its momentum is transferred to the metal phase and to the adjacent gas volume in the surroundings (Grant et al 1993). The decay of subsonic axial gas velocity from annular close-coupled atomizers has been investigated by laser doppler anemometry (LDA) and photographic methods (Bewlay and Cantor 1990; Grant et al 1993). The above studies have yielded velocity vector maps showing the variation in droplet velocities as a function of axial distance from the region of atomization. In addition, a variety of theoretical decay profiles of axial gas velocity have been used in numerical models of gas atomization (Mathur et al 1989; Gutierrez et al 1989; Eon-Sik Lee and Ahn 1994). In each case the results have been approximated to an exponential decay of the axial gas velocity with distance as illustrated in (1) (Eon-Sik Lee and Ahn 1994):

$$
\frac{u_{\mathrm{g}}(z)}{u_{\mathrm{o}}}=\left[1+\left(\frac{z}{\lambda}\right)^{20}\right]^{-0.05},
$$

where, $u_{\mathrm{g}}(z)$ is the axial gas velocity, $u_{\mathrm{o}}$ the initial gas velocity on exit from the atomizer, $z$ the axial distance from the point of atomization, $\lambda$ the exponential gas velocity decay coefficient, given by (2) below

$$
\lambda=\alpha \sqrt{A_{\mathrm{e}}},
$$

where, $\alpha$ is the empirically determined constant relating to kinematic viscosity of gas and is determined as 10.5 (Bewlay and Cantor 1989) and $A_{\mathrm{e}}$ the exit area of the atomizer. 
When a particle (assumed to be spherical) is accelerated or decelerated during its motion in a fluid, the statement of Newton's second law takes the following form of (3) (Szekely 1979)

$$
\begin{aligned}
\frac{4}{3} \prod R_{\mathrm{p}}^{3} \rho_{\mathrm{p}} \frac{\mathrm{d} U}{\mathrm{~d} t}= & +\frac{4}{3} \prod R_{\mathrm{p}}^{3}\left(\rho_{\mathrm{p}}-\rho\right) g \\
& (\text { force due to gravity) } \\
& +\frac{C_{\mathrm{d}} \Pi R_{\mathrm{p}}^{2}}{2} \rho U|U| \\
& \quad(\text { drag force) } \\
& -\tilde{C}_{\mathrm{A}} \frac{4}{3} \Pi R_{\mathrm{p}}^{3} \rho \frac{\mathrm{d} U}{\mathrm{~d} t} \\
& (\text { added mass effect) } \\
& -C_{\mathrm{H}} R_{\mathrm{p}}^{2}(\Pi \rho \mu)^{\frac{1}{2}} \int_{0}^{t} \frac{\mathrm{d} U}{\mathrm{~d} \tau} \frac{d \tau}{\sqrt{t-\tau}}, \\
& (\text { history term) }
\end{aligned}
$$

or

$$
\begin{aligned}
\rho_{\mathrm{d}} V_{\mathrm{d}} \frac{\mathrm{d} u_{\mathrm{d}}}{\mathrm{d} t}= & V_{\mathrm{d}}\left(\rho_{\mathrm{d}}-\rho_{\mathrm{g}}\right) g-\frac{1}{2} \rho_{\mathrm{g}} A_{\mathrm{d}} C_{\mathrm{d}}\left|u_{\mathrm{d}}-u_{\mathrm{g}}\right| \\
& \left(u_{\mathrm{d}}-u_{\mathrm{g}}\right) .
\end{aligned}
$$

Equation (4) indicates that only the inertia force, the gravitational force and the droplet drag force determine the droplet motion (Eon-Sik Lee and Ahn 1994). They are then subjected to a retarding drag force. The drag force is related to the droplet acceleration by Newton's second law (Geiger and Poirier 1973; Lavernia et al 1988). In (4), $C_{\mathrm{d}}$ is the drag coefficient, $\rho_{\mathrm{g}}$ the gas density; $\mathrm{d}, \mathrm{m}$ and $\mathrm{u}$ are droplet diameter, mass and velocity, respectively. The drag coefficient, $C_{\mathrm{d}}$, varies with the droplet Reynolds number,

$$
\operatorname{Re}=\left(\frac{\rho_{\mathrm{g}} U_{\mathrm{d}}}{\mu_{\mathrm{g}}}\right),
$$

where, $U=u_{\mathrm{g}}-u_{\mathrm{d}}$ is the gas/droplet slip velocity and $\mu_{\mathrm{g}}$ the gas velocity and can be approximated to within $7 \%$ over the range $0 \cdot 1<\mathrm{Re}<4000$ by (5) (Clift et al 1978). Equation (6) gives the relationship between drag coefficient and Reynold's number,

$$
C_{\mathrm{d}}=0 \cdot 28+\left(\frac{6 \sqrt{\operatorname{Re}}+21}{\operatorname{Re}}\right) .
$$

Equations (3) to (6) help in calculating the axial droplet velocities as a function of flight distance. It is obvious that there is no analytical solution to (4) (Mathur et al 1989). This equation is solved numerically at nodes by dividing the flight trajectory of the droplet into short segments over which the equation is assumed to be valid.
Recently Dimos and John (1997) have reported in their studies on heat transfer and fluid dynamics in spray deposition, that when compressible effects are important (Mach number of the gas is quite high), they should be accounted for in the drag coefficient expression. This indicates that (6) is not appropriate when supersonic exit velocities are involved. In such cases a correlation shown in (7) developed for rocket nozzles should be used (Crowe et al 1972),

$$
C_{\mathrm{d}}=\frac{24}{\operatorname{Re}}\left[\frac{\left(1+0 \cdot 15 \mathrm{Re}^{0.687}\right)\left(1+\exp \left[-\frac{0 \cdot 427}{M^{4 \cdot 63}}-\frac{3}{\mathrm{Re}^{0.88}}\right]\right)}{1+\frac{M}{\operatorname{Re}}\left[3 \cdot 82+1 \cdot 28 \exp \left(-\frac{1 \cdot 25 \mathrm{Re}}{M}\right)\right]}\right]
$$

where, $M$ is the Mach number and Re the Reynold's number both of which are based on the relative velocity between the particle and the gaseous stream and the gaseous stream properties.

\subsection{Droplet heat transfer}

The microstructures and in turn properties of sprayed alloy can be predicted to a considerable extent by calculating the droplet thermal histories (Gutierrez et al 1988; Lavernia et al 1988). The heat extraction from the droplets takes place by convective cooling because of a large temperature difference between molten metal droplets and cool atomizing gas. In this regard, the investigators have suggested that for liquid metal droplets during atomization convective cooling dominates over radiative cooling and hence radiation effects can be safely neglected (Lavernia et al 1988; Mathur et al 1989; Grant et al 1993; Eon-Sik Lee and Ahn 1994). However, since the heat extraction from a droplet surface depends on the relative velocity between the cooling gas and the droplet itself, it is necessary to estimate droplet and gas velocities as discussed by Lavernia et al (1988).

Most of the researchers have adopted lumped parameter models (LPM) for calculation of thermal histories (Lavernia et al 1988; Mathur et al 1989; Gutierrez et al 1989; Grant et al 1993; Eon-Sik Lee and Ahn 1994; Dimos and John 1997). Moreover, Levi and Mehrabian (1982) have successfully shown that LPM would give satisfactory results at large undercooling when temperature gradients within the melt reach very large values (Levi and Mehrabian 1982). The advantage with LPM is the simplicity of computation, since first order ordinary differential equations are to be solved. Since the size of the droplet is less in gas atomization, one can neglect the heat conduction within the droplets i.e. the droplet temperature is uniform (Lavernia et al 1988; Grant et al 1993; Eon-Sik Lee and Ahn 1994). In addition, the process of conduction freezing using LPM as well as radially sym- 
metric nonisothermal model has been analysed (Bayazitoglu and Cerny 1993). In addition to the radial symmetry imposed on the droplets, the presence of recalescence resulting from severe undercooling and the associated nonequilibrium phenomena was neglected. It has been found that at cooling rates of $10^{4} \mathrm{~K} / \mathrm{s}$, the LPM was sufficiently accurate and the assumption of constant temperature inside the droplets justified. This amounts to a well-known approximation of Newtonian cooling and gives rise to LPM.

A LPM of the quenching of a spherical liquid droplet can be constructed by equating the rate of change in surface of the sensible heat contained in the droplet to the rate of heat extraction through the outer surface of the droplet (Lavernia et al 1988),

$$
\rho_{\mathrm{m}} V_{\mathrm{d}} C_{\mathrm{pm}} \frac{\mathrm{d} T}{\mathrm{~d} t}=h\left(T-T_{0}\right) A_{\mathrm{d}} .
$$

Equation (8) takes the form of (9) for a small spherical droplet, where, $\rho_{\mathrm{m}}$ is the density of the metal, $C_{\mathrm{pm}}$ the specific heat of the metal, $V_{\mathrm{d}}$ the volume of the droplet equal to $4 \pi r_{\mathrm{d}}{ }^{3} / 3$ and $A_{\mathrm{d}}$ the area of the spherical droplet equal to $4 \pi r_{\mathrm{d}}^{2}$.

$$
\frac{\mathrm{d} T}{\mathrm{~d} t}=\frac{6 h\left(T-T_{0}\right)}{\rho_{\mathrm{m}} C_{\mathrm{pm}} \mathrm{d}} .
$$

Equation (8) or (9) can be readily integrated if heat transfer coefficient, $h$, is assumed to be constant. But in actual droplet gas interactions heat transfer coefficient cannot be a constant since the velocity of the gas decays and that of the droplet increases. Therefore, (10) and (11) are employed for time intervals small enough for the variation in $h$ to be infinitesimal.

$$
\begin{aligned}
& T=\left(T_{\mathrm{p}}-T_{\mathrm{o}}\right) e^{\left(\frac{-t}{\tau}\right)}+T_{\mathrm{o}}, \\
& \tau=\rho_{\mathrm{m}} C_{\mathrm{pm}}\left(\frac{V_{\mathrm{d}}}{A_{d}}\right) h .
\end{aligned}
$$

Since radiation effects can be neglected, the heat transfer coefficient ' $h$ ' is calculated using (12) (Ranz and Marshall 1952)

$$
h=\left[\frac{K_{\mathrm{g}}}{2 r_{\mathrm{d}}}\right]\left[2+0 \cdot 6 N_{\mathrm{Re}}^{1 / 2} N_{\mathrm{Pr}}^{1 / 3}\right],
$$

and

$$
\begin{aligned}
& N_{\mathrm{u}}=\left[2+0 \cdot 6 N_{\mathrm{Re}}^{1 / 2} N_{\mathrm{Pr}}^{1 / 3}\right], \\
& P_{\mathrm{r}}=\frac{C_{\mathrm{g}} \mu_{\mathrm{g}}}{K_{\mathrm{g}}},
\end{aligned}
$$

where, $C_{\mathrm{g}}$ is the gas specific heat, $\mu_{\mathrm{g}}$ the absolute viscosity of gas and $K_{\mathrm{g}}$ the gas thermal conductivity.
Equation (13) represents Nusselt number given by well known (Ranz and Marshall 1952) correlation for laminar convection from a solid sphere. The Nusselt number is defined on the basis of the droplet diameter, the surfaceaveraged heat transfer coefficient between the gas and the droplet and the free-stream thermal conductivity. The Prandtl number is that of the gas at free-stream conditions. The Reynold's number is based on the relative velocity between the droplets and the free-stream.

Here it is important to note that the Ranz and Marshall correlation used by many researchers (Lavernia et al 1988; Mathur et al 1989; Grant et al 1993; Eon-Sik Lee and Ahn 1994) has limited validity (Dimos and John 1997). This is because it is well established that Ranz and Marshall correlation for ' $h$ ' is appropriate when the Reynold's number lies in the range of $0 \cdot 1$ to 4000 . In the cases of supersonic gas atomization Reynold's number exceeds 4000. In such a situation it was felt that Whitaker's (1972) correlation for heat transfer from an isothermal spherical surface is more accurate:

$$
N_{\mathrm{u}}=\frac{h D}{K}=2+\left(0 \cdot 4 \operatorname{Re}^{1 / 2}+0 \cdot 06 \operatorname{Re}^{2 / 3}\right) \operatorname{Pr}^{0.4}\left(\frac{\mu_{\infty}}{\mu_{\mathrm{s}}}\right)^{1 / 4},
$$

and

$$
h=\left[\frac{K_{\mathrm{g}}}{2 r_{\mathrm{d}}}\right]\left[2+\left(0 \cdot 4 \mathrm{Re}^{1 / 2}+0 \cdot 06 \operatorname{Re}^{2 / 3}\right) \operatorname{Pr}^{0.4}\left(\frac{\mu_{\infty}}{\mu_{\mathrm{s}}}\right)^{1 / 4}\right],
$$

where, $K_{\mathrm{g}}$ is the thermal conductivity of the gas, Re the Reynold's number and Pr the Prandtl number. Equation (16) is recommended for $3.5<\operatorname{Re}<7.6 \times 10^{4}, 0.71<$ $\operatorname{Pr}<380$ and $1<\mu_{\infty} / \mu_{\mathrm{s}}<3 \cdot 2$.

\subsection{Droplet solid fraction and secondary dendrite arm spacing}

It is important to know the fraction solidified and the dendrite arm spacing in the droplets during flight from the practical point of view (Gutierrez et al 1989). During solidification, since the temperature of the droplet is assumed to remain unchanged (Newtonian cooling) and radiation is neglected, the energy balance yields (17) (Bing and Lavernia 1999),

$$
\frac{\mathrm{d} f}{\mathrm{~d} t}=\frac{6}{H_{\mathrm{f}} \rho_{\mathrm{d}} D} \frac{k_{\mathrm{g}}}{D}\left(T-T_{0}\right)\left[2+0 \cdot 6 N_{\mathrm{Re}}^{1 / 2} N_{\mathrm{Pr}}^{1 / 3}\right] .
$$

But (17) has to be modified if the Reynold's number exceeds 4000. Equation (18) shows the modified energy balance equation incorporating Whitaker's correlation 


$$
\begin{aligned}
\frac{\mathrm{d} f}{\mathrm{~d} t}= & \frac{6}{H_{\mathrm{f}} \rho_{\mathrm{d}} D} \frac{k_{\mathrm{g}}}{D}\left(T-T_{0}\right) \\
& {\left[2+\left(0 \cdot 4 \operatorname{Re}^{1 / 2}+0 \cdot 06 \operatorname{Re}^{2 / 3}\right) \operatorname{Pr}^{0.4}\left(\frac{\mu_{\infty}}{\mu_{\mathrm{s}}}\right)^{1 / 4}\right], }
\end{aligned}
$$

where, $\mathrm{d} f$ is the solidification fraction, $H_{\mathrm{f}}$ the heat of fusion, $\rho_{\mathrm{d}}$ the droplet density, $V_{\mathrm{d}}$ the droplet volume and $D$ the droplet diameter. It has been reported that the fraction solid monotonically increases as a function of flight distance (Gutierrez et al 1989). This is because the droplet solidifies on cooling through melting range. The relationship established between secondary dendrite arm spacing and cooling rate can be adopted for computing the DAS in the flying droplet (Flemings 1974). Equation (19) can be used to predict the size scale of the microstructure of the as-solidified droplets.

$$
\lambda=a T^{\mathrm{b}},
$$

where, $\lambda$ is the SDAS in micrometers, $T$ the cooling rate in ${ }^{\circ} \mathrm{C} / \mathrm{s}, a$ and $b$ are empirical constants.

It has been found that the initial $10 \%$ of solid phase forms very rapidly, at a distance of about $0.2 \mathrm{~m}$ during recalescence following nucleation. Further, the cell size is nearly independent of fraction solidified and hence the flight distance. Therefore, cell size in the droplets, which solidify in flight, depends mostly on the droplet diameter (Gutierrez et al 1989).

During the present investigation, a comprehensive general-purpose software code was developed to take care of computations as per mathematical modeling. Figure 1 shows the flow chart for the same. The code is adaptable to any alloy system with given parameters in case of supersonic gas atomization. Table 1 shows the input parameters used for computations.

\section{Experimental}

In the present investigation, trials were planned with Al$\mathrm{Si}-\mathrm{Mg}$ (LM 25/A356). The atomization was carried out using a supersonic convergent-divergent nozzle designed to develop $3 \mathrm{Mach}$ at $0.4 \mathrm{MPa}$ atomizing pressure. The atomizer assembly consisted of a gas inlet chamber and concentric collar to protect the metal delivery tube from chilling. The metal delivery tube was made of stainless steel with inner diameter $0.006 \mathrm{~m}$ and was attached to a bottom pouring type stainless steel crucible. The crucible was maintained at pouring temperature of the alloy in order to avoid hot shortness. The protrusion length of metal delivery tube was adjusted to $0.003 \mathrm{~m}$. The alloy was melted in an electrical resistance furnace. Specified melting practice was followed with degasification using hexachloroethane tablets. Nitrogen was used as the atomizing gas. Some modifications (See et al 1973) were done on the fabricated spray casting experimental set-up for the purpose of droplets/powder collection. The substrate platform was replaced with a water trough as shown in figure 2 . The droplets could be quenched and collected in water trough placed at a stand off distance of $1 \mathrm{~m}$. This stand off distance was selected to ensure the physical state of most of the droplets to be solid. In actual spray deposition process the stand off distance will be much less to capture mushy droplets at the substrate. One of the objectives of the study was to suggest a stand off distance, which results in maximum yield as well as microstructural refinement.

Collected droplets are dried and sieved to classify them to various size fractions using standard sieve set. Subsequently, droplet sizes of 50, 100, 200, 400 and $500 \mu \mathrm{m}$ were selected for investigation of dynamic and thermal behaviour. These droplets were cold mounted and prepared for microexamination. Optical photomicrographs were

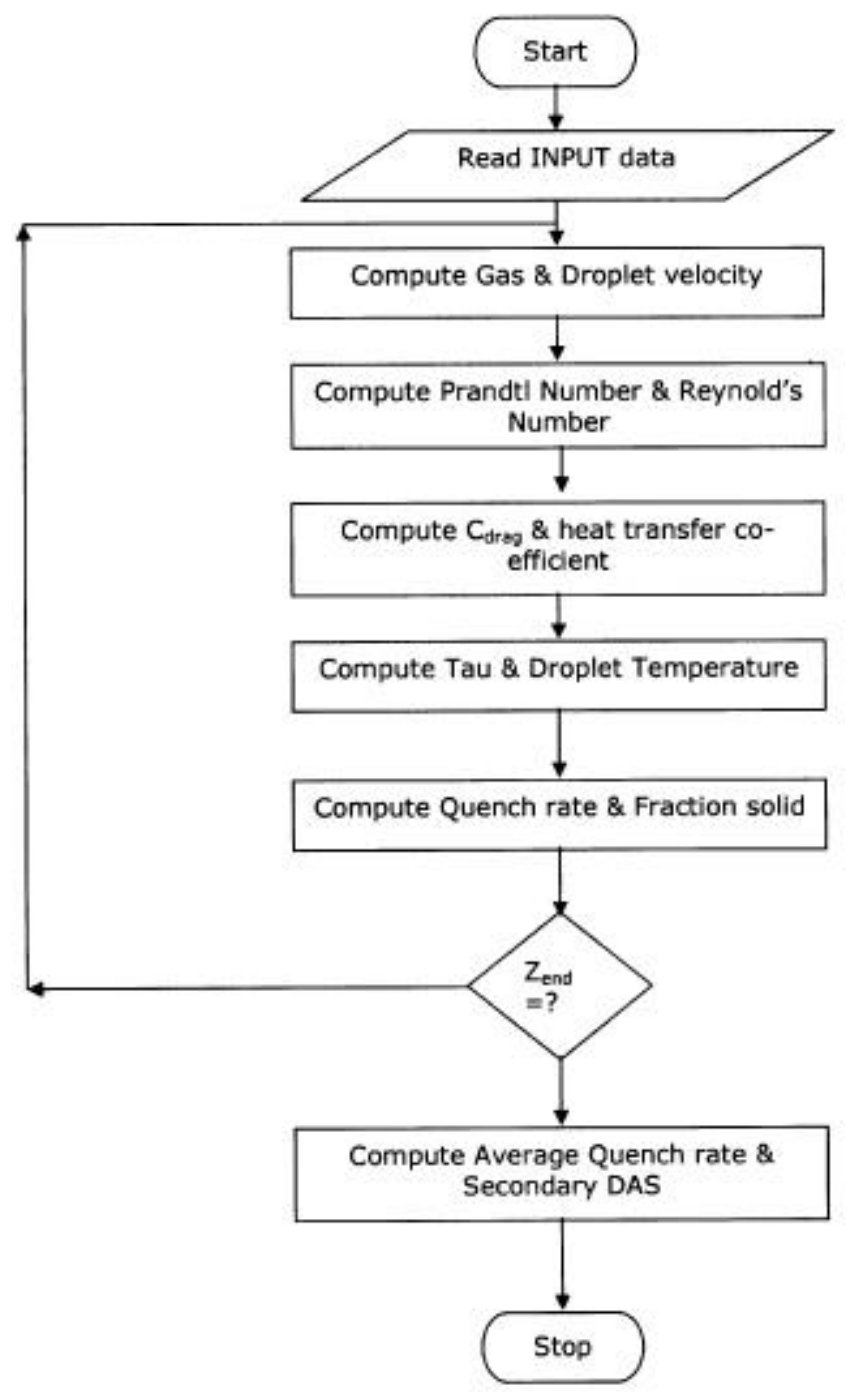

Figure 1. Flow chart for modeling of droplet dynamic and thermal behaviour. 
obtained at different magnifications using Nikon Epiphot optical microscope. Figures 11 and 12 show the microstructure of two extreme sizes of the droplets/powder particles. Further, secondary dendrite arm spacing was measured using quantitative metallographic technique.

\section{Results and discussion}

\subsection{Dynamic behaviour}

In the present investigation, the nozzle was designed to develop Mach no. 3. The gas velocity decay from the exit of the nozzle was computed. Figure 3 shows the velocity profile as a function of flight distance. Initial gas velocity was taken as $1000 \mathrm{~m} / \mathrm{s}$, which was the designed exit velocity. From the figure it can be seen that the gas velocity decays exponentially as a function of flight distance and reaches $200 \mathrm{~m} / \mathrm{s}$ at a distance of $0.7 \mathrm{~m}$ from the region of atomization. This alone could not provide information on the impact velocity of the droplets. Nevertheless, this was the first step for computing droplet velocity. In the present investigation, instead of considering a constant velocity (or average velocity), instantaneous gas velocity obtained from gas velocity plot was used for obtaining droplet velocity profile for variously sized droplets. This should provide more realistic droplet velocity and augments the accuracy of the model.

Figure 4 shows the Reynolds number variation as a function of flight distance for different droplet sizes computed based on the relative velocity of the gas and droplet. From the figure it is seen that the Reynold's number for the larger droplets is more as anticipated. This is obvious because the Reynold's number is directly propo-

Table 1. Input data for the software code indicating properties of Al-6 wt\% Si alloy and nitrogen gas.

\begin{tabular}{rll}
\hline \multicolumn{1}{l}{$\begin{array}{l}\text { S1. } \\
\text { no. }\end{array}$} & Quantity & Value \\
\hline 1. & Density of gas $\left(\rho_{\mathrm{g}}\right)$ & $1.25 \mathrm{~kg} / \mathrm{m}^{3}$ \\
2. & Density of droplet $\left(\rho_{\mathrm{d}}\right)$ & $2400 \mathrm{~kg} / \mathrm{m}^{3}$ \\
3. & Velocity of gas $\left(V_{\mathrm{g}}\right)($ Mach No. 3) & $1000 \mathrm{~m} / \mathrm{s}$ \\
4. & Velocity of droplet $\left(V_{\mathrm{d}}\right)$ & $10 \mathrm{~m} / \mathrm{s}$ \\
5. & Viscosity of gas $\left(\mu_{\mathrm{g}}\right)$ & $1 \cdot 7 \times 10^{-5} \mathrm{~kg} / \mathrm{m}-\mathrm{s}$ \\
6. & Exit area of atomizer $\left(A_{\mathrm{e}}\right)$ & $302 \cdot 986 \times 10^{-6} \mathrm{~m}^{2}$ \\
7. & Constant depending on & $10 \cdot 5$ \\
& kinematic viscosity of gas $(\alpha)$ & \\
8. & Temperature of molten metal $\left(T_{\mathrm{p}}\right)$ & $1123 \mathrm{~K}$ \\
9. & Temperature of atomizing gas $\left(T_{0}\right)$ & $277 \mathrm{~K}$ \\
10. & Thermal conductivity $\left(K_{\mathrm{g}}\right)$ & $2 \cdot 6 \times 10^{-3} \mathrm{~W} / \mathrm{m}-\mathrm{K}$ \\
11. & Specific heat of gas $\left(C_{\mathrm{p}}\right)$ & $1040 \mathrm{~J} / \mathrm{kg}-\mathrm{K}$ \\
12. & Specific heat of metal $\left(C_{\mathrm{pm}}\right)$ & $850 \mathrm{~J} / \mathrm{kg}-\mathrm{K}$ \\
13. & Empirical constants for DAS $(a)$ & $45 \mu \mathrm{m}$ \\
14. & Empirical constants for DAS $(b)$ & $-0 \cdot 25$ \\
15. & Flight distance $(z)$ & $1 \mathrm{~m}$ \\
16. & Liquidus temperature $\left(T_{\mathrm{l}}\right)$ & $847 \mathrm{~K}$ \\
17. & Solidus temperature $\left(T_{\mathrm{s}}\right)$ & $673 \mathrm{~K}$ \\
\hline
\end{tabular}

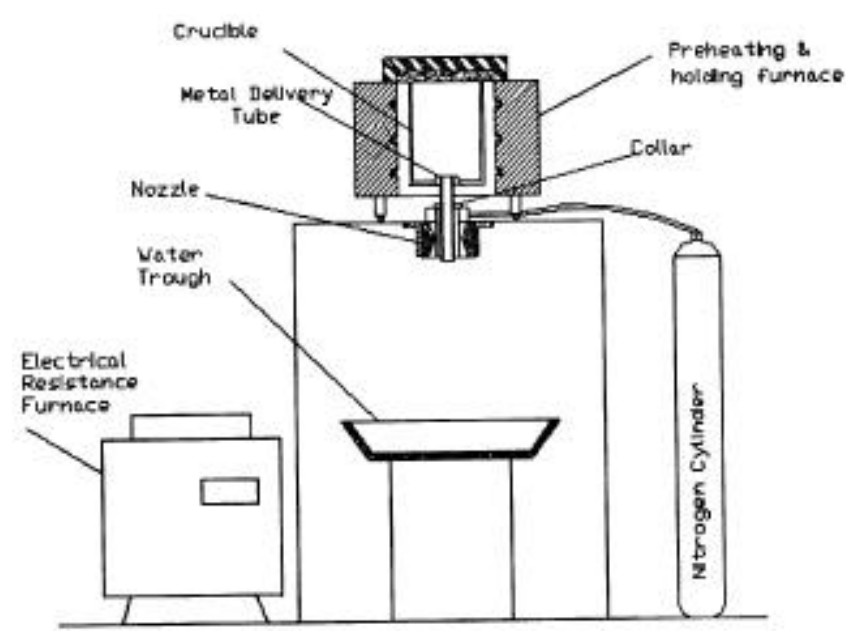

Figure 2. Experimental setup used for droplet collection.

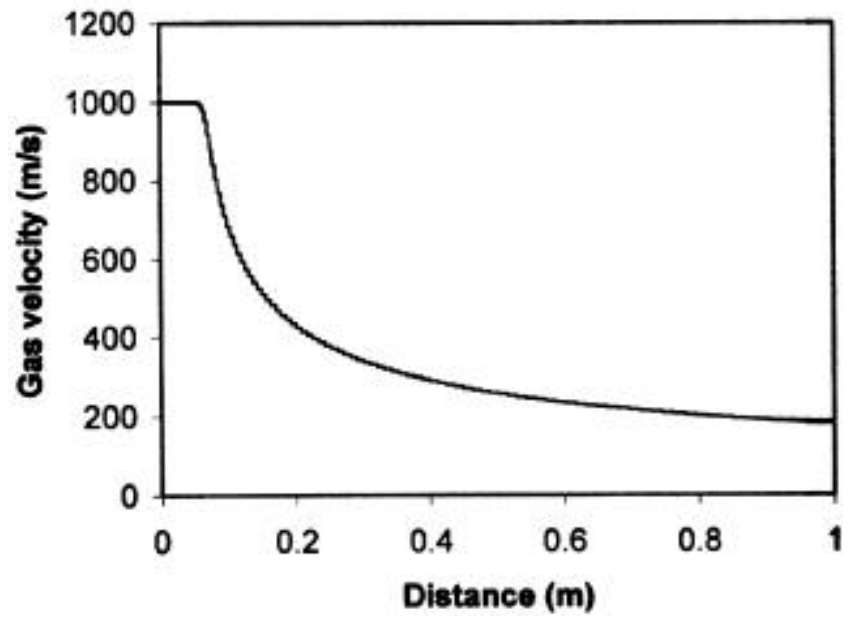

Figure 3. Variation of gas velocity as a function of flight distance.

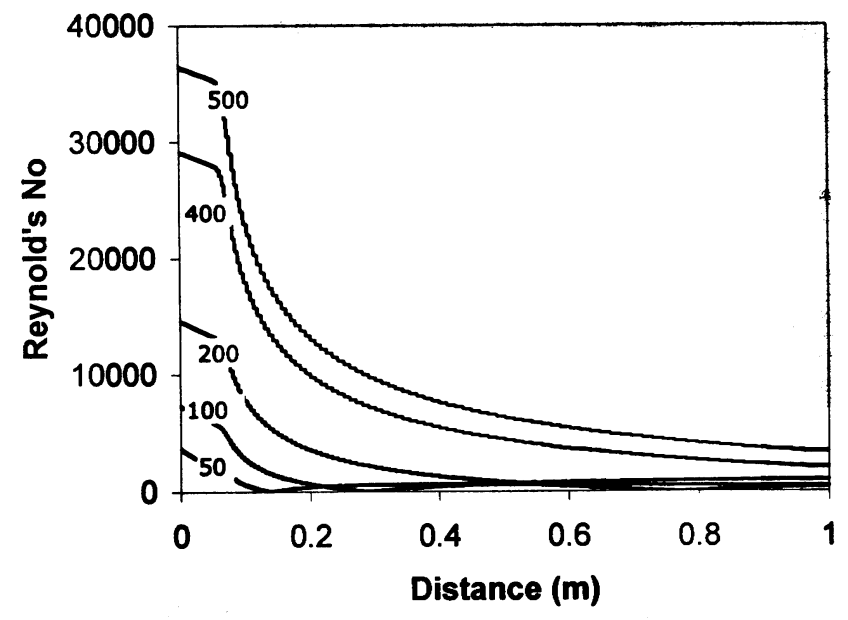

Figure 4. Variation of Reynold's number for different droplet sizes $(\mu \mathrm{m})$ as a function of flight distance. 
rtional to the droplet diameter. It is very significant to note that the Reynold's number for all the chosen droplet sizes was exceeding 4000 in the present study.

Further, the Reynold's number plot was used to compute drag coefficient $\left(C_{\mathrm{d}}\right)$, which was required to calculate droplet velocities. Figure 5 shows the drag coefficient (representing opposing force to motion of droplets) plot for different droplet sizes. Here care was taken to use appropriate correlation for $C_{\mathrm{d}}$ since higher Mach number (and Reynold's number) was involved. In this connection, drag coefficient relations for high-speed flow past a small sphere developed from research related to rocket nozzle was adopted wherein Mach number in addition to Reynold's number term is present. From the figure it is clear that the drag coefficient reaches a maximum value and decreases to become asymptotic to the $\mathrm{x}$-axis for all the droplet sizes under consideration. This is attributable to the variation in the Reynold's number for different droplet sizes. The Reynold's number decreases to zero during the flight since the relative velocity between the droplet and the atomizing gas becomes zero.

Figure 6 illustrates the droplet velocity profile for variously sized droplets along with gas velocity profile. From the figure it is seen that at the exit of the nozzle the droplets have least velocity (equivalent to acceleration due to gravity) and during the flight they gain velocity owing to the momentum transfer from the atomizing gas. The smallest droplet would be attaining highest velocity during the flight and vice versa. The relative velocity of gas and droplets becomes zero as the flight distance increases and is analogous to Reynold's number variation. The smaller droplets are attaining maximum velocity at a shorter time and subsequently they travel faster than atomizing gas. Reynold's number for smaller droplets are extremely low and in such cases the drag forces are significantly large. As a result smaller droplets are decelerating faster and show noticeable peak velocity. On the

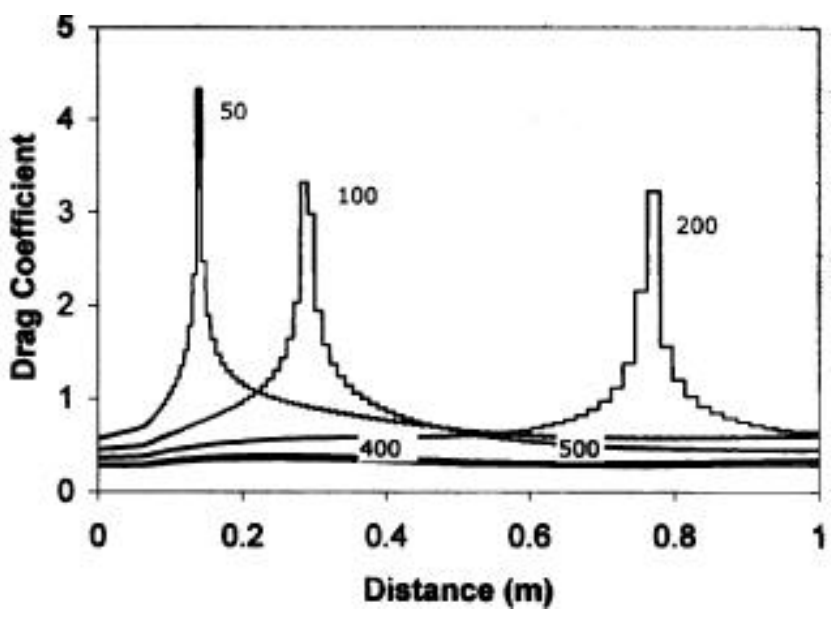

Figure 5. Variation of drag coefficient of various droplet sizes $(\mu \mathrm{m})$ as a function of flight distance. contrary, the drag coefficient for the larger droplets is approximately constant after the peak velocity owing to their high inertia force and hence they do not show a significant deceleration. It was also noticed that most of the droplets are at considerably high velocity $(>100 \mathrm{~m} / \mathrm{s})$ at the time of impingement on the substrate for a stand off distance of $0.6 \mathrm{~m}$. This aspect is critical to address the microstructural evolution in the spray cast alloys. Many investigators have proposed dendrite fragmentation mechanism to explain equiaxed grain morphology since mushy (semi-solid/semi-liquid) droplets reach the substrate with considerable velocity. In the present investigation, there was certainly a possibility for dendrite fragmentation as evident from the droplet velocity plots. However, thermal/ physical state of the individual droplets are to be considered to determine which droplet size would be in mushy state to undergo dendrite fragmentation at the time of impact. The next section deals with the thermal histories of droplets based on their size.

\subsection{Thermal behaviour}

First step in predicting the thermal state of different droplets was to obtain the heat transfer coefficient as a function of flight distance. In this regard, Whitaker's correlation for heat transfer from isothermal spherical surface was incorporated in the software code to obtain heat transfer coefficient plot. From figure 7 it is clear that the smaller droplets would have larger heat transfer rate since they have larger surface area to volume ratio. The instantaneous heat transfer coefficient was used for obtaining thermal history of droplets.

Figure 8 shows thermal history profile for different droplet sizes as a function of flight distance. The solidus and liquidus temperatures are illustrated for the alloy under consideration. This was done to identify the physi-

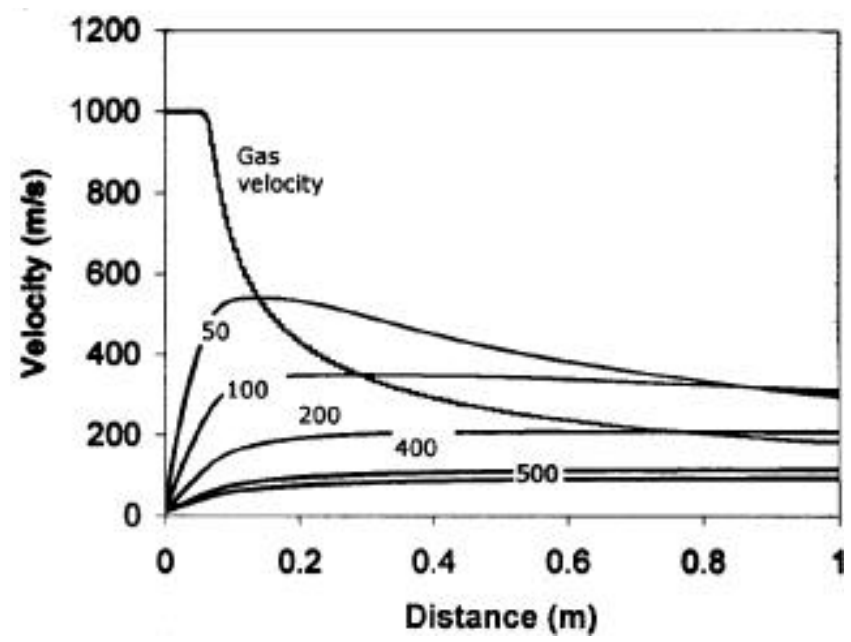

Figure 6. Variation of gas velocity and droplet velocities as a function of flight distance. 
cal state of the droplets based on their size when they reach the substrate. It is well recorded that mushy droplets are most desirable to obtain the best quality preform. It was necessary to determine which size range of the droplets reach the substrate in mushy state. A better way to quantify this was to determine the solid fraction of the droplets as a function of flight distance.

Figure 9 shows the variation of percentage solid or solid fraction of the droplets of various sizes during their flight. This plot was obtained by plugging in the instantaneous heat transfer coefficient (from figure 7), which varies as a function of gas velocity along the flight distance.

From figure 9 it can be seen that the smaller droplets become completely solidified at a distance of 0.2 to $0.6 \mathrm{~m}$. The droplets of more than $100 \mu \mathrm{m}$ sizes would be in semi solid/semi liquid state for a longer time or a greater flight distance. An important outcome of the above analysis was optimization of one of the critical parameters viz. standoff distance for given set of atomization parameters and a particular alloy system. In the light of above, a standoff distance of 0.6 to $0.7 \mathrm{~m}$ was recommended for obtaining preforms in $\mathrm{Al}-\mathrm{Si}-\mathrm{Mg}$ alloy during the deposition trials. At this stand off distance it was estimated that the droplets of size range between 100 and $500 \mu \mathrm{m}$ possess $90 \%$ to $15 \%$ solid fraction respectively. Whereas the droplets smaller than this size reach the substrate as solid powders, which may either be lost as overspray $(<10 \mu \mathrm{m}$ size), bounce off (if the preform surface has solid layer), or may get entrapped in the preform (if the preform has a mushy/liquid layer). On the other hand droplets of larger than $500 \mu \mathrm{m}$ diameter reach the substrate in liquid state resulting in splattering. The parameters in spray casting trials should be controlled in such a way that the spray has more volume fraction of mushy droplets i.e. the droplets of size around $200-300 \mu \mathrm{m}$.

Figure 10 shows the cooling rates prevailing in droplets. This illustrated rapid solidification effects and was

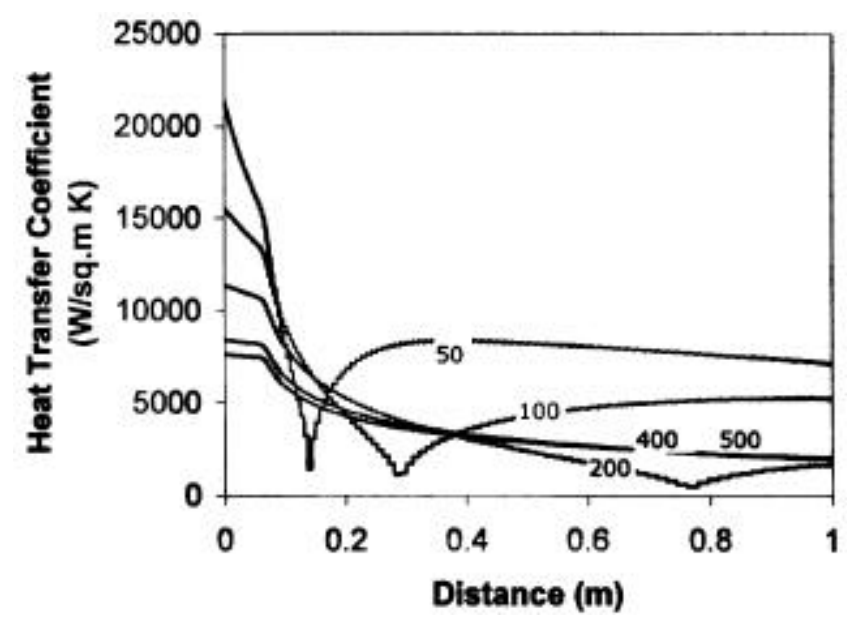

Figure 7. Variation of heat transfer coefficient of different droplet sizes $(\mu \mathrm{m})$ as a function of flight distance.

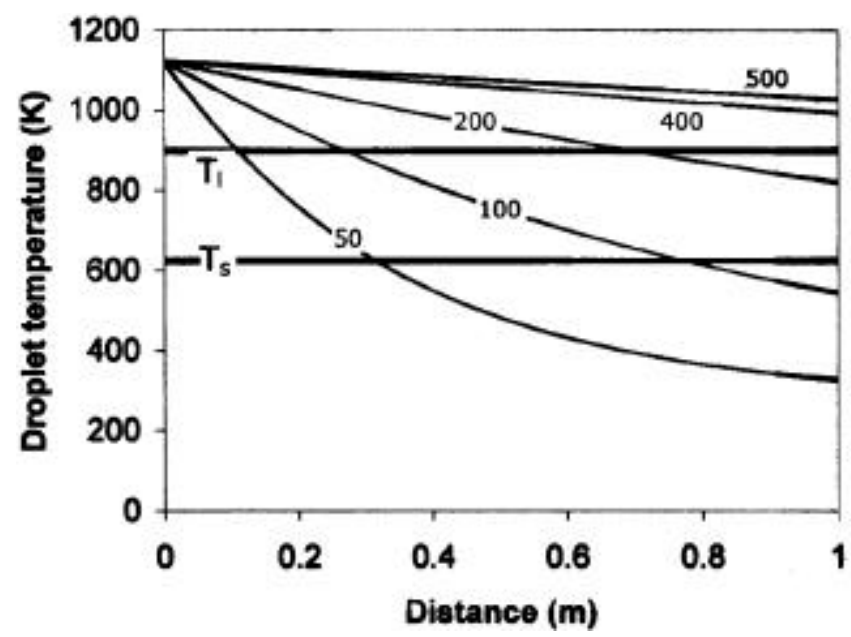

Figure 8. Thermal history of droplets of different droplet sizes $(\mu \mathrm{m})$ as a function of flight distance; $T_{1}$ and $T_{\mathrm{s}}$ are liquidus and solidus temperatures, respectively.

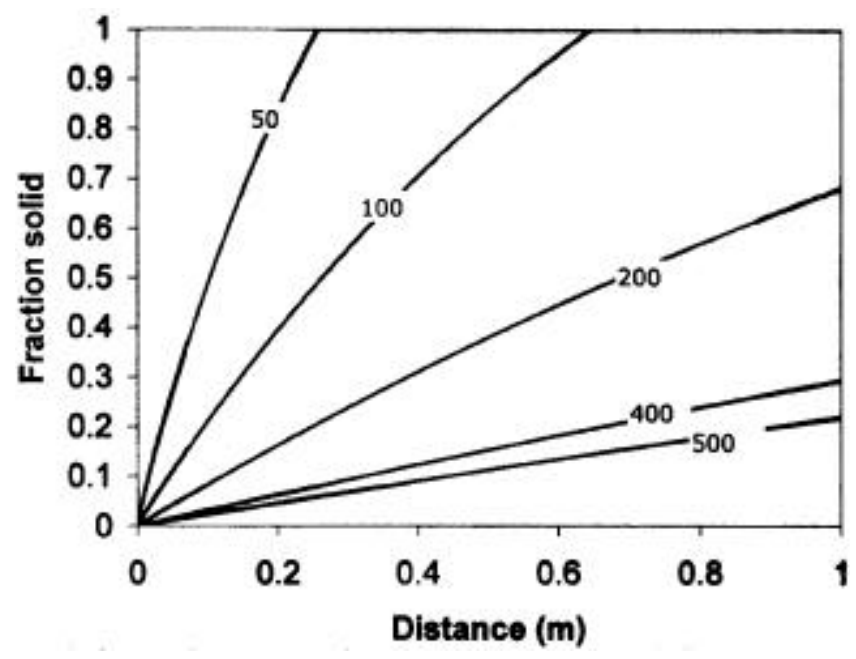

Figure 9. Fraction solid of different droplet sizes $(\mu \mathrm{m})$ as a function of flight distance.

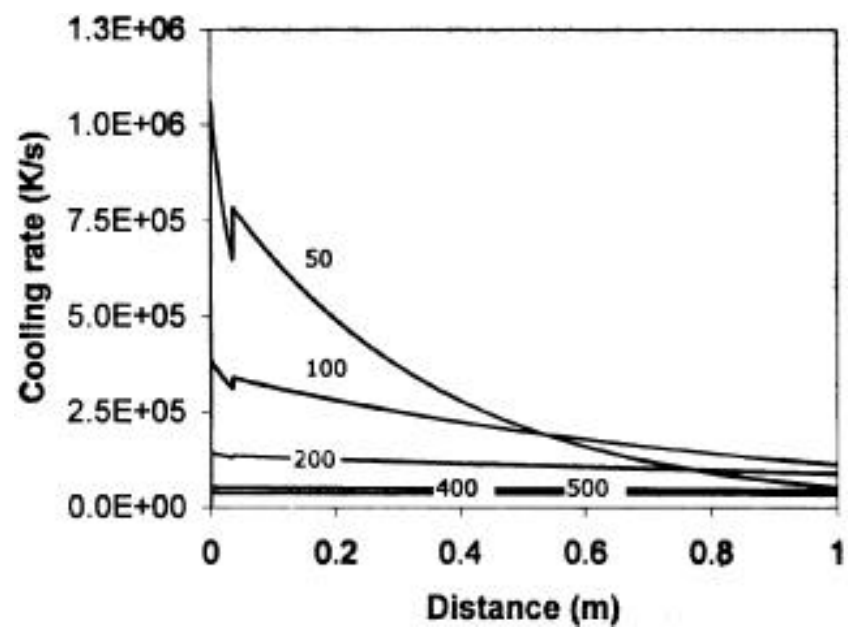

Figure 10. Cooling rate of different droplet sizes $(\mu \mathrm{m})$ as a function of flight distance. 
ultimately useful to plot secondary dendrite arm spacing curve. From the figure it could be noticed that for most of the droplet sizes the cooling rate was found to be of the order of $10^{5} \mathrm{~K} / \mathrm{s}$. This could confirm the rapid solidification effects during atomization. Cooling/quenching rate for smaller droplets were more since they lose heat faster. The cooling rate values were used to compute secondary dendrite arm spacing for different droplet sizes.

Figure 11 shows the optical micrograph of powder of $50 \mu \mathrm{m}$ size at $1000 \times$ magnification. It shows fine dendrite structure with average secondary dendrite arm spacing of around $1 \cdot 1 \mu \mathrm{m}$. Figure 12 shows the micrograph of $500 \mu \mathrm{m}$ powder at $400 \times$ magnification. Randomly grown dendrite arms can be seen indicating the presence of a number of nucleation sites within a droplet. The secondary arm spacing was coarser and was found to be around $4 \mu \mathrm{m}$. This size of the droplet would be in mushy state while reaching the substrate. Such droplets with veloci-

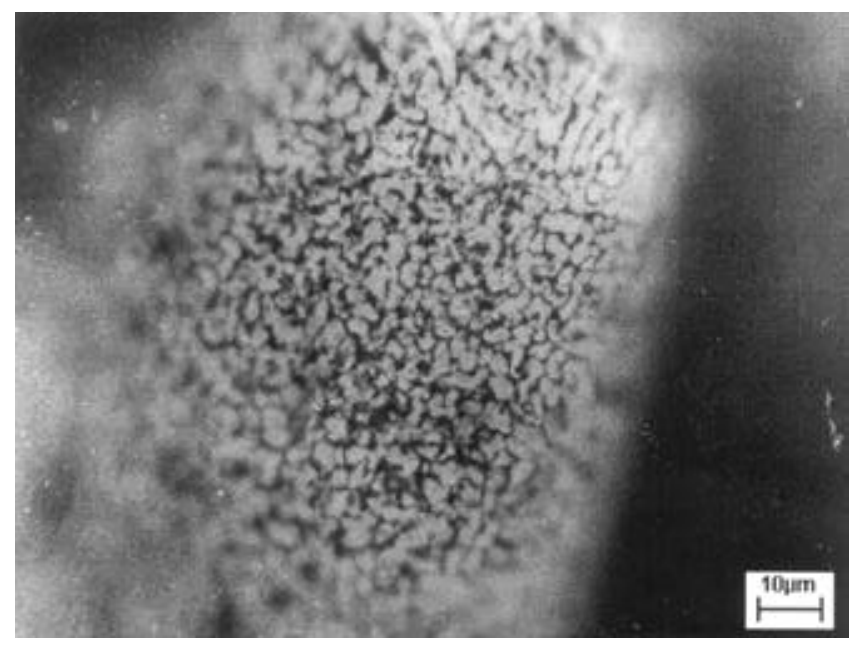

Figure 11. Microstructure of $50 \mu \mathrm{m}$ powder showing SDAS of $1.1 \mu \mathrm{m}$.

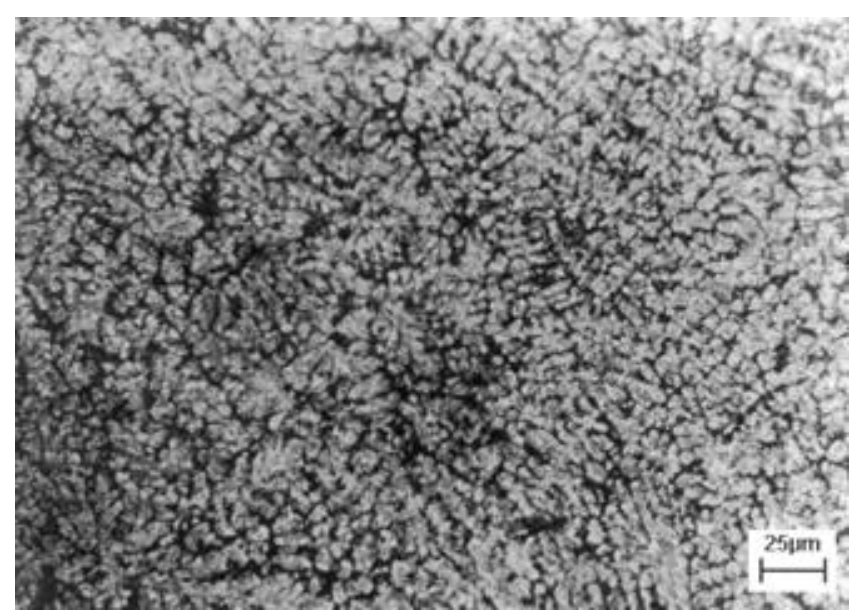

Figure 12. Microstructure of $500 \mu \mathrm{m}$ powder showing SDAS of $4 \mu \mathrm{m}$. ties higher than $100 \mathrm{~m} / \mathrm{s}$ would impact the top surface of the substrate and undergo dendrite fragmentation. Figure 13 shows the comparison of predicted and experimentally obtained SDAS values. The predicted SDAS were close to measured SDAS for all the droplet sizes under consideration. Moreover, the mean droplet size of $200 \mu \mathrm{m}$ showed much closer proximity to the experimental SDAS.

\section{Conclusions}

(I) Supersonic conditions were given due consideration during the mathematical modeling of droplet dynamic and thermal behaviour. This was necessary because the nozzle was designed to develop $3 \mathrm{Mach}$. In this regard, the correlations used for finding drag coefficient and heat transfer coefficients were particularly suitable for higher Reynold's number existing in the present situation.

(II) From droplet velocity computation, it was found that most of the droplets impinge the substrate with considerably high velocity. This would initiate dendrite fragmentation and hence an increase in nucleation density. The above observation supported the evolution of equiaxed grains due to the dendrite fragmentation.

(III) Thermal history and fraction solid plot for various droplet sizes indicated the physical state of the droplet corresponding to their sizes. It was found that the droplet sizes beyond $100 \mu \mathrm{m}$ are in mushy state as they reach the substrate. In addition, optimization of stand off distance was possible based on the fraction solid at a particular distance. This optimal distance should be maintained during the spray deposition by retracting the substrate at a specified rate analogous to the deposition rate.

(IV) Cooling rate of individual droplet size was computed and in turn secondary dendrite arm spacing was determined. The predicted SDAS values were reasonably in good agreement with experimentally determined values.

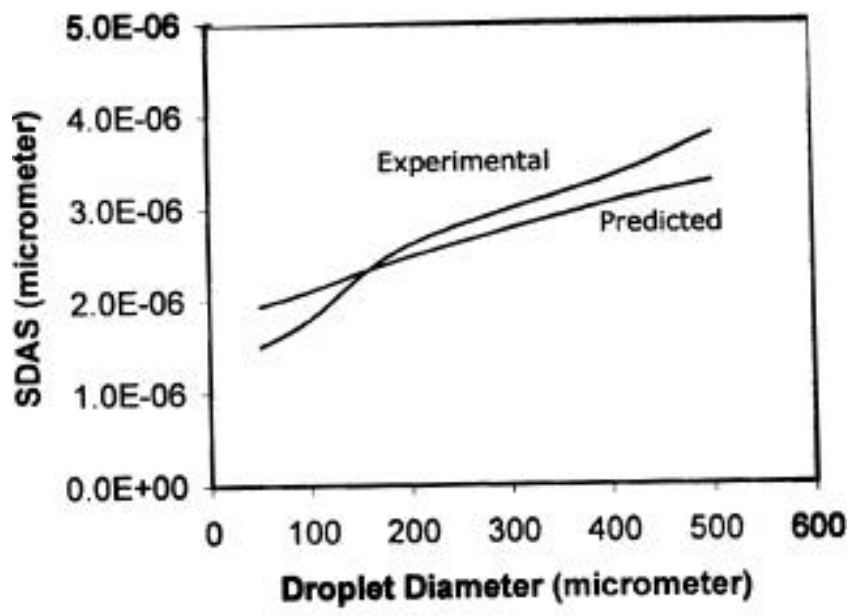

Figure 13. Comparison of predicted and experimental values of SDAS for different droplet sizes. 


\section{Acknowledgements}

The authors are grateful to the Foundry and Forge Division and the Central Materials and Processes Laboratory, Hindustan Aeronautics Ltd., Bangalore, for their technical support. Our special thanks to Dr Kishore T Kashyap and Mr T Chandrashekar, M.S. Ramaiah Institute of Technology, Bangalore, for fruitful discussion.

\section{References}

Anderson I E, Figliola R S and Morton H 1991 Mater. Sci. Eng. A148 101

Bayazitoglu Y and Cerny R 1993 J. Mater. Process. Manuf. Sci. 251

Bewlay B P and Cantor B 1989 Mater. Sci. Eng. A118 207

Bewlay B P and Cantor B 1990 Met. Trans. B21 819

Bing Li and Lavernia E J 1999 in Spray-forming; Non-equilibrium processing of materials (ed.) C Suryanarayana (UK: Pergamon Materials Series) p.153

Clift R, Grace J R and Weber M E 1978 Bubbles, drops and particles (NY: Academic Press) p. 111

Dimos Poulikakos and John M Waldvogel 1997 Adv. Heat Trans. 281

Eon-Sik Lee and Ahn S 1994 Acta Metall. 423231

Flemings M C 1974 Solidification processing (NY: McGrawHill) pp 34 and 149

Geiger G H and Poirier D R 1973 Transport phenomena in metallurgy (Reading, Massachusetts: Addison-Wesley) pp 68 and 157

Grant P S 1995 Spray-forming, in Progress in materials science (UK: Pergamon Press) 39 p. 497

Grant P S and Cantor B 1991 Cast Metals 4140

Grant P S and Cantor B 1995 Acta Metall. 43913
Grant P S, Cantor B and Katgerman L 1993 Acta Metall. 41 3097, 3109

Gutierrez E M, Lavernia E J, Trapaga G M and Szekely J 1988 Int. J. Rapid Solidification 4125

Gutierrez E M, Lavernia E J, Trapaga G M, Szekely J and Grant N J 1989 Met. Trans. A20 71

Lavernia E J 1989 Int. J. Rapid Solidification 547

Lavernia E J, Gutierrez E M, Szekely J and Grant N J 1988 Int. J. Rapid Solidification 489

Lawley A 2000 in Melt atomization and spray deposition-Quo Vadis, Proceedings of international conference on spray deposition and melt atomization (eds) $\mathrm{K}$ Bauckhage, $\mathrm{V}$ Uhlenwinkel and U Fritsching (Bremen, Germany: University of Bremen) 1 p. 3

Levi C J and Mehrabian R 1982 Met. Trans. A13 221

Lubanska H 1970 J. Metals 2245

Mathur P, Apelian D and Lawley A 1989 Acta Metall. 37429

Ojha S N 1992 Bull. Mater. Sci. 24527

Perepezko J H and Park J S 1982 Rapidly solidified amorphous and crystalline alloys (eds) B H Kear, B C Giessen and M Cohen (NY: North Holland) p. 49

Ranz W E and Marshall W R 1952 Chem. Eng. Prog. 48 141, 173

See J B, Runkle J and King T B 1973 Metall. Trans. 4 2669, 2673

Szekely J 1979 Fluid flow phenomena in metals processing (NY: Academic Press) pp 337 and 261

Unal A 1987 Mater. Sci. \& Technol. 31029

Unal A 1988 Mater. Sci. \& Technol. 4909

Whitaker S 1972 AlChE J. 18361

Xu Q and Lavernia E J 2000 in Fundamentals of the spray forming process, Proceedings of international conference on spray deposition and melt atomization (eds) $\mathrm{K}$ Bauckhage, V Uhlenwinkel and U Fritsching (Bremen, Germany: University of Bremen) 1 p. 17 